\title{
Droits de propriété en économie pastorale. Le raisonnement hardinien est-il crédible?
}

The hardinien reasoning: is it credible enough for the upholders of "property

rights" in pastoral economy?

\section{Adil Roumane}

\section{(2) OpenEdition}

\section{Journals}

Édition électronique

URL : http://journals.openedition.org/economierurale/3926

DOI : 10.4000/economierurale.3926

ISSN : 2105-2581

\section{Éditeur}

Société Française d'Économie Rurale (SFER)

Édition imprimée

Date de publication : 15 mai 2013

Pagination : 21-33

ISSN : 0013-0559

Référence électronique

Adil Roumane, «Droits de propriété en économie pastorale. Le raisonnement hardinien est-il crédible ? », Économie rurale [En ligne], 335 | mai-juin 2013, mis en ligne le 15 mai 2015, consulté le 21 avril 2019. URL : http://journals.openedition.org/economierurale/3926 ; DOI : 10.4000/economierurale.3926 


\section{Droits de propriété en économie pastorale Le raisonnement hardinien est-il crédible?}

Adil ROUMANE • Centre d'Études sur la Mondialisation, les Conflits, les Territoires et les Vulnérabilités » (CEMOTEV-E.A. 4457), Université de Versailles Saint-Quentin-en-Yvelines (UVSQ)

Les débats autour de l'allocation optimale des ressources forestières ont mis en évidence la forte dépendance entre leur soutenabilité et les régimes de propriété. Pour privilégier la solution privative, les tenants de l'École des droits de propriété ont d'abord opté pour la remise en cause de la propriété commune qu'ils considèrent comme facteur accélérant la détérioration de la ressource. Si leurs arguments sont multiples, le plus célèbre reste l'article de Hardin relatif à la tragédie des biens communs. L'auteur propose d'imaginer une communauté d'éleveurs partageant un pré en commun. Tenté par la maximisation de sa propre utilité, chaque éleveur continuera d'élargir la taille de son troupeau pour augmenter son rendement, ce qui se traduira par une détérioration tragique de la ressource. La solution est donc de privatiser le pré. L'auteur tentera de soumettre ce raisonnement à la réalité pour évaluer sa crédibilité.

MOTS-CLÉS : Hardin, droits de propriété, foresterie, propriété commune, privatisation

\section{The hardinien reasoning: is it credible enough for the upholders of "property rights" in pastoral economy?}

The debate goshawks of the optimal allowance of forest resources brought to light the fort dependence between their sustainability and the property regimes. To privilege the privative solution, the upholders of the "property rights" opted at first for the depreciation of the common property considered as factor which accelerates the deterioration of the resource. If their arguments are multiple, the most famous is Hardin's article relative to the tragedy of the commons. The author suggests imagining a breeder's community who shares and exploits a meadow. To maximize his own utility, every breeder will continue to widen the size of his herd to increase his return, what will be translated by a tragic deterioration of the resource. According to Hardin, the only solution is the privatization of the meadow. We would try to examine this reasoning in reality in the purpose of estimate its credibility. (JEL: Q23, Q27, Q57).

KEYWORDS: Hardin, property rights, forestry, common property, privatization

$\mathbf{S}^{\mathrm{i}}$ l'économie relative au monde rural s'intéresse le plus souvent à l'agriculture, la foresterie n'y est pas moins importante, notamment dans les pays pauvres où le lien entre le stock disponible et le développement socioéconomique est particulièrement fort. Les activités pastorales sont un bon exemple. Dans ce contexte, les débats autours de l'allocation optimale des ressources forestières en général et fourragères en particulier ont mis en évidence la forte dépendance entre les modes d'exploitation et les régimes de propriété. Avec l'émergence de la notion d'effets externes (Pigou, 1920) et la montée en puissance de l'École des droits de propriété (Coase, 1960 ; Dales, 1968) les systèmes communautaires de gestion seront vigoureusement mis en cause dès le milieu du siècle précédent. Ces mises en cause se sont appuyées dans un premier temps sur les conclusions de Warming (1911), sur les modèles de Gordon (1954) et de Scott (1955) expliquant la diminution de la rente 
tirée des pêcheries par l'accès libre, puis sur les conclusions de Garrett Hardin dans son célèbre article de 1968 sur la tragédie des communaux. Hardin, tout comme ses prédécesseurs, s'en est pris à la propriété commune des parcours pour conclure que leur dégradation ne peut s'expliquer que par l'absence de droits de propriété qu'implique ce régime. Les économistes appartenant à l'École des droits de propriété déprécient explicitement toute appropriation communautaire qu'ils considèrent comme à l'origine des dommages infligés aux actifs environnementaux. Ce raisonnement s'appuie sur les deux caractéristiques qui lui ont été accordées, à savoir la forte rivalité et l'absence d'exclusion. D'après les tenants de ce courant, c'est l'utilité individuelle qui prime systématiquement en cas de propriété commune au détriment de l'intérêt général et du bien-être collectif. Chaque exploitant sera ainsi tenté par la maximisation de sa propre utilité qui entraînera forcément la surextraction de la ressource sans aucune prise en compte des coûts sociaux qui en résultent (Alchian, Demsetz, 1973). La solution optimale réside donc dans l'appropriation de ces ressources, soit d'une manière privative ou étatique.

L'objectif de ce travail est d'évaluer jusqu'à quel point le modèle théorique de Hardin est crédible pour servir d'argument justifiant la dépréciation de la propriété commune afin de célébrer la solution privative. Pour mener à bien notre réflexion, nous entamerons notre débat par un aperçu global sur le lien entre la soutenabilité des ressources pastorales et les différents modes de leur gestion. Nous exposerons dans un deuxième temps les lacunes du raisonnement hardinien, puis nous exposerons les limites qui peuvent être celles de la propriété privée sur les ressources fourragères. Nous tenterons par la suite de justifier notre point de vue par une représentation graphique illustrant les faiblesses du principal exemple auquel la tragedy of the commons se réfère. En conclusion, nous tenterons de mettre en lumière le rôle que peuvent jouer les structures traditionnelles locales dans la régulation de l'activité pastorale.

\section{Soutenabilité des ressources pastorales face aux modes de gestion}

Les débats autour de l'allocation optimale des ressources forestières en général et pastorales en particulier ont mis en évidence la forte dépendance entre les modes de gestion et les régimes de propriété. Plusieurs auteurs ont confirmé ce constat à l'instar de Wibe (1992) pour qui «les multiples fonctions de la gestion forestière sont étroitement liées aux questions de régime de propriété et de réglementation ». Avec le recul sans précédent des massifs forestiers dans la plupart des pays en développement, les activités pastorales ont vigoureusement été mises en cause comme facteurs de déforestation. Cette situation a relancé les discours sur le système de gestion le plus optimal permettant de garantir un stock fourrager constant et de maintenir un rendement non décroissant dans le temps. Ce débat a ravivé la controverse qui a traditionnellement opposé les tenants de la gestion communautaire à ceux de la solution privative d'une part, ainsi qu'à ceux de l'interventionnisme étatique d'autre part.

Mais dans cette optique, il est à noter que les expériences de domanialité déjà adoptées par plusieurs pays en développement n'ont pas donné les résultats escomptés. Bien au contraire, elles confirmaient l'échec de l'étatisme ainsi que l'inefficience des traditions régaliennes en foresterie. De nombreuses études empiriques ont montré jusqu'à quel point le déclin des systèmes traditionnels de gestion en faveur d'une gestion dite administratisée s'est traduit par une régression constante des surfaces boisées (Roumane et al., 2010). Et c'est face à ce constat que la gestion 
des ressources forestières va prendre une nouvelle tournure à la suite de la prise en compte du coût d'opportunité qui résulte de l'exclusion des populations locales et de la perte qui en découle en termes de stock. Il sera ainsi admis que l'échec des stratégies de gestion forestière s'explique en partie par la non prise en compte des pratiques coutumières adaptées aux spécificités socioculturelles locales. Les principes d'une gestion forestière dite communautaire ou participative vont progressivement trouver leur place dans la littérature (Anaya, 1996 ; Booth, 1998 ; Buchy, Hoverman, 2000 ; Chouinard, Perron, 2002 ; Varughese, Ostrom, 2001). Les acteurs scientifiques, les représentants des ONG et des organisations internationales vont conclure que l'implication des tribus usagères dans le processus de gestion, la reconnaissance de leurs pratiques et de leurs traditions permettront d'inverser la tendance en limitant les rythmes préoccupants de déforestation particulièrement sous les tropiques (Colfer, Resosudarmo 2002 ; Kiss 1990 ; Brown 1999).

Néanmoins, l'approche communautaire en foresterie n'est pas sans antagonistes. Plusieurs réflexions aussi bien normatives que positives menées dans le champ de l'économie l'ont remise en cause au point même de la considérer comme facteur entraînant une dégradation irréversible de la ressource. Cette optique regroupe, en particuliers, les économistes appartenant à l'École des droits de propriété qui considèrent l'appropriation communautaire comme à l'origine des dommages infligés à la ressource par l'exploitation non soutenable à laquelle il donne naissance. Ils affirment qu'en l'absence d'un propriétaire bien défini, c'est l'utilité individuelle qui prime systématiquement au détriment de l'intérêt général et du bienêtre collectif. Aucune règle à prendre en compte n'existe. Chaque exploitant, tenté par la maximisation de sa propre utilité, opte pour un usage rationnel sans aucune prise en compte des coûts sociaux qui peuvent en résulter (Alchian, Demsetz, 1973). La solution optimale réside dans l'appropriation de la ressource soit d'une manière privative soit étatique. Les fidèles de ce courant manifestent leur préférence envers la solution privative en s'appuyant sur la thèse malthusienne quand aux effets néfastes des populations sur les ressources, et en reflétant l'esprit de Smith quant à la rationalité individuelle qui caractérise les agents économiques.

C'est donc cette conception utilitariste qui justifie l'appel à la privatisation en considérant que «la nature se porte mieux lorsque les gens, cuvrant dans le secteur privé, se consacrent à la poursuite de leur intérêt individuel, dans un contexte où ils sont tenus responsables de leurs actions, mais où ils sont également libres d'innover»(Philippe 2007). Cela signifie que sans propriétaire privé ayant la possibilité d'exercer son droit d' " exclusion » au sens de Samuelson (1954), l'actif naturel risque d'être voué à l'épuisement. En admettant ce raisonnement, le terme «propriété commune »n'indique pas une forme de propriété mais plutôt une situation de non propriété caractérisée par l'accès libre où les règles d'usage sont mal ou non définies (Gordon, 1954 ; Scott, 1955 ; Demsetz, 1967 ; Alchian, Demsetz, 1973). Ces deux derniers sont même allés plus loin, jusqu'à dire que la possession des droits communaux peut inciter les individus à avoir des comportements malhonnêtes. Mais, l'argument le plus réputé reste le célèbre article de Hardin (1968) relatif à la «tragédie des communs ». L'idée phare développée est que toute activité pastorale basée sur une exploitation commune se traduira systématiquement par une dégradation tragique de la ressource. Et c'est justement dans cette optique où notre réflexion s'inscrit en essayant de répondre à la question suivante : jusqu'à quel point peut-on considérer le 
modèle de Hardin comme cadre référentiel standard duquel nous pouvons tirer une conclusion définitive?

\section{La réplique au fondement théorique de la tradition hardinienne}

Avant d'entamer l'analyse et la critique des arguments hardiniens, plusieurs remarques sont à signaler : premièrement la réflexion de Hardin, qui est celle d'un biologiste, est loin d'être compatible avec le raisonnement d'un économiste. Deuxièmement, l'expérience moyenâgeuse sur laquelle l'exemple hardinien repose, reste largement dépassée par le temps et inadaptable au contexte actuel. Troisièmement, l'exclusivité spatiale de l'analyse (l'Angleterre) dont les spécificités biogéographiques et socioéconomiques ne peuvent en aucun cas être les mêmes dans toutes les régions du globe. Cette inadaptabilité spatiotemporelle ne permet pas, et par principe, la généralisation de la doctrine hardinienne qui reste purement imaginaire, puisqu'elle ne s'appuie sur aucune étude empirique, concrète et bien localisée. Rien ne justifie, par conséquent, la légitimité de l'universalité absolue qui lui a toujours été accordée.

Mais, l'erreur historique de Hardin réside dans le sens qu'il a accordé à la propriété commune. Il s'agit d'une assimilation erronée de cette dernière au libre accès pour annoncer la prééminence et l'efficacité de l'appropriation privative. Chez Hardin et ses successeurs, c'est la propriété commune qui est devenue opposée à la propriété privée au lieu de la propriété publique ! La problématique de conservation des ressources naturelles ne peut pas être réduite à la simple et seule question du régime de propriété comme si c'était le statut juridique de la ressource qui favorisait sa dégradation. Curieusement, les partisans de la tradition hardinienne, et plus généralement les tenants de la théorie des droits de propriété, ignorent la possibilité de dégradation d'une ressource privatisée comme si c'était son statut privé qui empêchait sa dégradation, et comme si aucune ressource privée n'avait jamais été dégradée ou même détériorée.

En outre, l'universalisme ainsi que le pessimisme hardinien n'ont pas épargné les liens intracommunautaires. Si les membres de la collectivité imaginée par Hardin sont tous individualistes, égoïstes et malveillants, rien n'empêche l'existence d'une communauté dont les membres seraient solidaires, altruistes et bienveillants. La conscience communautaire envers l'intérêt général, et malgré l'importance primordiale qu'elle revêt, a toujours été négligée par les fidèles de la tradition hardinienne. Les multiples travaux qui ont été menés dans le champ de la sociologie prouvent que la sociabilité, la solidarité et l'entraide ont toujours caractérisé la vie communautaire des populations locales en milieu rural.

Le raisonnement hardinien laisse bien comprendre que les ressources en propriété commune sont dénuées de propriétaire bien défini, en rapprochant la propriété commune de l'absence de propriétaire. S'il s'appuie sur le critère de rivalité (qui reste probable et pas inéluctable) pour assimiler la propriété communautaire à l'accès libre, il ignore complètement le critère d'exclusion qui caractérise le plus souvent la propriété commune en l'assimilant à la propriété privée. Dans le cas de plusieurs collectivités, le droit d'usage est catégoriquement limité aux exploitants locaux. Ces derniers s'attribuent le droit d'exclure tout individu non appartenant à leur tribu. Vivien (2002) affirme dans cette optique que : «Propriété commune et absence de propriété recouvrent des situations très différentes. Une communauté d'individus peut très bien s'organiser pour gérer une ressource naturelle; des droits sont 
conférés à chacun des membres du groupe détenteur de la ressource et des mécanismes institutionnels locaux permettent d'exclure les individus n'appartenant pas au groupe d'usagers. Ainsi, de nombreuses études de cas montrent qu'une gestion $d u$ rable des ressources peut exister sans qu'il $y$ ait une structure juridique de droit privé ou de droit étatique. »(Ostrom, 1990). Lorsqu'il s'agit d'une ressource possédée en commun, l'exclusivité est conditionnée par l'abondance de la ressource. Plus cette dernière est rare, plus la tribu est rigoureuse envers l'exclusion des exploitants étrangers. Il est à noter que ces derniers sont, dans la plupart des cas, exclus même lorsqu'il s'agit d'une ressource commune relativement abondante. De ce fait, on peut noter que la propriété commune n'est pas un statut juridique totalement homogène. Compte tenu des règles, des droits et d'obligations qui font parfois autorité à l'intérieur de la communauté usagère, on peut donc faire la distinction entre une propriété commune régulée et une propriété commune non régulée. Si la deuxième est plutôt proche de la propriété publique (et est visiblement celle qui est considérée par l'exemple de Hardin), la première peut être considérée comme une propriété quasi privée ou même une propriété privée partagée.

L'une des lacunes des partisans de l'École des droits de propriété, c'est qu'ils rejettent catégoriquement l'idée de l'efficacité de la gestion communautaire alors que les expériences démontrant le contraire se sont multipliées à travers l'histoire. Dans plusieurs cas où la ressource est possédée en commun, des règles coutumières d'exploitation et d'usage sont appliquées sous l'égide des organisations traditionnelles locales. Cet avantage permet d'éviter toute situation tragique au sens de Hardin. La bonne application des normes communautaires endogènes permet de lutter contre les comportements opportunistes et les formes d'exploitation illégale en combinant les valeurs d'équité sociale et les enjeux d'une gestion participative (nous développerons ce point au chapitre suivant). Le modèle de Hardin (1968) montre bien les dangers de l'accès libre sans préciser que le problème réside plutôt dans la non définition des ayants-droits que dans le régime de propriété lui-même. Si cette définition est garantie en cas d'une propriété privée, elle peut l'être également lorsqu'il s'agit d'une propriété commune. Dans un ouvrage plus récent publié en (1994), Hardin lui-même s'est employé à rectifier cette lacune en faisant une distinction bien claire entre les terres communes non aménagées et les terres communes aménagées où les droits de propriété permettent d'éviter l'usage impropre de la ressource (Mc Kean, Ostrom, 1995). Si Hardin luimême a varié sur ses propres analyses de la propriété commune, on peut se demander comment les tenants de l'École des droits de propriété s'appuient toujours sur des arguments contestés et rectifiés par leur propre auteur?

\section{Quelle efficacité pour la solution hardinienne?}

Hardin a proposé comme solution de renoncer à la propriété commune pour proposer la privatisation du pré via sa répartition entre les éleveurs. Mais jusqu'à quel niveau cette solution peut-elle être considérée comme efficiente?

Premièrement, l'acceptabilité sociale de la solution se pose comme un véritable problème. Rien ne garantit que tous les bergers accepteront de diviser le pré, et rien ne justifie d'imposer à certains d'entre eux cette solution qui ne les arrange pas juste parce qu'elle arrange leurs voisins. Même au cas où la solution du morcellement est acceptée par l'ensemble des usagers, l'équité de la répartition se pose 
comme problème supplémentaire compte tenu de la variabilité des rendements localisés. Si le stock fourrager est relativement le même sur la surface du pré (ce qui n'est pas toujours le cas), cela ne semble pas être le cas pour les massifs forestiers notamment en zones semi-arides où la strate herbacée est quasi absente, et où la répartition spatiale de la flore présente de grandes disparités. Chaque berger a donc intérêt à posséder la zone la plus productive, et cela au détriment du berger le moins favorisé qui obtiendra par conséquent la zone la plus dégradée. Un autre point faible réside dans l'éloignement de la parcelle obtenue par rapport à la zone d'habitation (village), ce qui complique le déplacement des troupeaux. Les bergers qui auront les zones les plus lointaines seront plus défavorisés par rapport aux bergers bénéficiant des zones les plus proches. Le coût et le temps de déplacement seront donc plus élevés pour les premiers. L'activité pastorale deviendra pour eux plus compliquée et plus difficile à exercer, la surveillance et le contrôle deviennent donc plus pénibles et plus coûteux ce qui est incompatible avec les principes de l'équité sociale.

En revanche, si le découpage de la surface se fait selon le nombre de bergers comme critère de répartition, les familles qui ne possèdent pas de troupeau n'auront donc pas droit à une partie de la forêt sachant que l'exploitation de cette dernière ne se limite pas seulement au pâturage. En outre, si le découpage se fait selon le nombre de foyers, ces derniers ne contiennent pas le même nombre d'individus, et n'ont donc pas le même niveau de besoins et ne possèdent pas la même taille de troupeau. Les familles nombreuses disposent de troupeaux plus grands compte tenu de leurs besoins plus importants, et de la disponibilité des personnes chargées de l'activité contrairement aux familles moins nombreuses. Il n'est donc pas raisonnable d'accorder la même surface à tous les types de familles.
À noter également que cette solution de morcèlement impliquera certainement la réduction de la surface totale du pré ou de la forêt après la fermeture des parcelles individuelles. Pour que chaque berger puisse accéder à sa parcelle sans passer par celle de son voisin, il reste nécessaire d'établir des passages et des pistes au milieu de la forêt. La surface totale des pistes sera certainement réalisée au détriment de la surface boisée qui réduira forcément la part et le bien-être de chacun et deviendra par conséquent une source d'effets externes. Ce morcellement portera nécessairement atteinte à l'équilibre de l'écosystème qui doit rester, par principe, une unité homogène. La faune sauvage y est en effet la première victime. Les limites matérielles définissant les bordures de chaque propriété réduisent les dimensions des habitats et limitent la mobilité des animaux. En outre, et vu la nécessité de séparer les parcelles par des barrières, et compte tenu du coût élevé de clôture par des barbelés ou outils similaires d'une part, et la pauvreté des usagers d'autre part, ces derniers recourent aux moyens traditionnels de clôture basés principalement sur l'usage des branches et des rameaux. Les travaux de clôture deviennent par conséquent un véritable facteur de déboisement. De surcroît, en dépit de toutes ces faiblesses, il est à noter que la soumission du raisonnement hardinien (lui-même) à la réalité laisse apparaître ses limites.

\section{L'explication graphique}

Concernant l'aspect tragique conféré par Hardin à l'exploitation de la ressource commune, il est à noter que la diminution du fourrage n'est pas forcément le résultat du seul facteur de surpâturage. Plusieurs autres facteurs peuvent l'influencer particulièrement le déficit pluviométrique et 
la longueur de la durée de sécheresse qui caractérisent plusieurs régions du globe. Dans ces zones, ce sont ces deux paramètres climatiques qui expliquent la diminution de la flore et la baisse du stock fourrager. Cette diminution n'implique aucun aspect tragique puisque le pâturage se rétablit dès la prochaine période pluvieuse au même titre que les arbres qui perdent leur feuillage dès l'arrivée de l'automne. Ceci est notamment le cas de plusieurs pays de la zone méditerranéenne où les caractéristiques biogéographiques restent totalement différentes de celles de l'Angleterre. La disparition de la strate herbacée est un phénomène tout à fait normal suivant la succession saisonnière, et qui est loin d'être tragique. L'herbe disparaît dès le début de la période estivale avec ou sans la présence des troupeaux. Il s'agit donc d'un phénomène tout à fait normal auquel les populations locales se sont adaptées à travers l'histoire et sans aucune situation « hardinement» tragique.

En outre, et même pour les régions où la succession saisonnière n'influence pas forcément le stock fourrager, le graphique suivant permet de contester le raisonnement hardinien. Soit E les effectifs des moutons et $\mathrm{Q}$ la quantité disponible en fourrage forestier. La droite OL désigne la rentabilité tirée du pâturage.

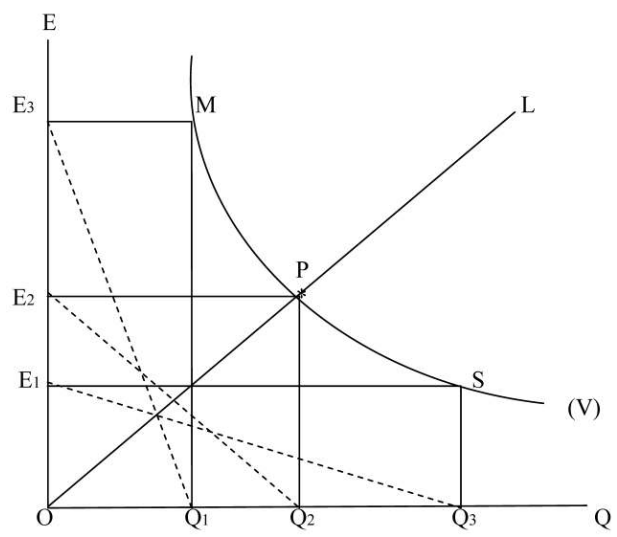

Si l'accroissement des effectifs de moutons dépend du stock fourrager disponible, ce dernier reste (suivant le raisonnement hardinien) variable en fonction de la taille des troupeaux comme le désigne la courbe (V). De ce fait, si l'accroissement des effectifs se traduit par la diminution $\mathrm{du}$ fourrage, leur diminution favorise par conséquent son abondance.

Soit E1, la taille minimale des troupeaux qui se traduit par une pression faible sur la ressource. Cela permet au stock fourrager d'atteindre son niveau maximum (Q3), ce qui donne un premier point d'équilibre (S). La surface (E1, S, Q3) correspond à l'aire au sein de laquelle l'accroissement du cheptel est sans répercussion sur la ressource commune. L'abondance du fourrage accorde au troupeau un accroissement jusqu' au niveau E2. Cet accroissement progressif des troupeaux réduit graduellement le stock du fourrage jusqu'à ce qu'il se situe au niveau Q2. Nous obtiendrons donc le point d'optimum (P) qui correspond au Rendement Maximal Soutenable (RMS) du pâturage au-delà duquel l'accroissement des troupeaux n'est plus souhaitable. La surface (E2, P, Q2) correspond donc à l'aire au-delà de laquelle l'accroissement des troupeaux n'est plus sans incidences.

Si l'accroissement du troupeau continue (soit le passage de E2 à E3), la diminution du stock fourrager sera par conséquent progressive jusqu'à ce qu'il atteigne son niveau minimal Q1. Au fur et à mesure, (en passant de Q2 à Q1) l'activité pastorale devient plus pénible et plus coûteuse et l'accroissement du cheptel ne devient plus rentable. Chaque bête supplémentaire se traduira par un coût supplémentaire. L'incertitude quant aux bénéfices qu'elle peut procurer reste néanmoins forte. Le troupeau atteindra par conséquent son niveau maximal au-delà duquel son accroissement ne sera plus possible soit le niveau (E3). On obtient donc un nouveau point d'équilibre soit $\mathrm{M}$ sur le graphique. La surface 
(E3, M, Q1) correspond donc à l'aire au sein de laquelle l'accroissement des troupeaux n'est plus rentable, voire même impossible. Cette diminution du fourrage ne peut se traduire que par la chute des effectifs des moutons. La pression sera donc faible, ce qui permet le renouvellement du stock fourrager et le retour au point initial d'équilibre soit (S) sur le graphique.

\section{L'aspect tragique du raisonnement hardinien ?}

Il est à noter que la diminution du fourrage se fait d'une manière plutôt progressive que brutale, ce qui fait que le passage du point d'équilibre initial (S) ou de l'optimum $(\mathrm{P})$ à la situation tragique au sens de Hardin ne se fait pas du jour au lendemain sans passer par une période que l'on peut qualifier de période de crise (soit le passage de la taille E2 à E3 et du stock Q2 à Q1). Cette période dont le point d'équilibre maximal se limite à $(\mathrm{M})$ sépare le point d'optimum (S) de la situation « hardiniennement » tragique de zéro fourrage. Plus on se dirige vers Q1, plus le fourrage diminue jusqu'à ce qu'on obtienne le point d'équilibre maximal (M). Le stock fourrager devient minimal mais pas nul.

Cette diminution ne favorisera pas l'augmentation du cheptel (qui atteindra son niveau maximal E3) ; au contraire, elle se traduira par la chute des effectifs et la réduction de la taille du troupeau avant d'atteindre la situation tragique de zéro fourrage. Au-delà du point d'équilibre (M), l'investissement supplémentaire des bergers n'est plus rentable compte tenu de la rareté du fourrage et de l'activité qui deviennent plus coûteuses et plus pénibles (déplacement, recherche du fourrage). Dans cette situation, il n'est plus favorable pour le berger d'augmenter la taille du troupeau puisque chaque unité supplémentaire implique un coût supplémentaire (peut-être plus élevé que le bénéfice qu'elle peut procurer), ce qui augmente le coût total et réduit la rentabilité. À partir de ce niveau, c'est le maintien de la taille du cheptel, voire même sa diminution, qui deviennent plus rentables que son augmentation.

Il est à noter que les composantes de la nature ont toujours fonctionné selon cet équilibre biologique notamment entre les effectifs des prédateurs et ceux des proies dont l'exemple le plus célèbre reste celui des lapins et des renards. L'accroissement des effectifs des prédateurs (les moutons dans l'exemple de Hardin) dépend de ceux des proies. Tout au long de l'histoire de l'évolution, les composantes de la biodiversité ont évolué selon cet équilibre sans que les prédateurs possèdent un droit de propriété ou de quotas sur les proies. Dans l'exemple de Hardin, les effectifs des moutons vont s'accrô̂tre jusqu'au niveau où le pâturage ne sera plus suffisant. Cette insuffisance se traduira, d'une part, par la réduction des effectifs, et d'autre part, par la minimisation de la pression qui permettra la régénération et la restitution du pâturage. On obtient donc le retour à la situation d'équilibre initial représentée sur le graphique par le point (S).

En réalité, l'activité d'élevage n'est pas un objectif en soi. Elle a toujours été accompagnée de l'exploitation du cheptel soit par la consommation soit par la commercialisation. Aucun berger n'a donc intérêt à supporter éternellement le coût de son activité sans en tirer du profit. En outre, les expériences montrent qu'à partir d'un certain âge, cette exploitation devient indispensable étant donné que la rentabilité de chaque bête diminue par rapport à son âge (sa vieillesse). Cette exploitation se traduira naturellement par la diminution des effectifs. Il est à noter également que les externalités négatives issues de l'exploitation des ressources naturelles sont, dans la plupart des cas, différentes de 
celles issues des rejets de polluants. Dans le cas de notre étude, l'externalité issue de l'accroissement du cheptel affectera le profit du berger lui-même, ce qui le pousse à modifier ou améliorer ses choix, contrairement à la firme polluante dont les bénéfices ne sont pas forcément affectés par sa propre pollution.

De ce fait, et « face aux défaillances de la propriété privée et de l'État, la gestion en commun réapparaît comme une solution et ce d'autant plus que son inefficience présumée dans la lignée de Hardin est due à une interprétation abusive de la notion de « commun » s'appuyant sur une confusion entre l'absence de propriété et la propriété collective (Ciriacy-Wantrup, Bishop, 1975). La tragédie des communaux s'exerce dans le cas d'absence de droits de propriété mais pas nécessairement dans celui de propriété commune. La propriété commune, contrairement à l'absence de propriété, se réfère à un ensemble de règles définies en lien avec l'appartenance des membres à une communauté. Baland et Platteau (1996) font une distinction entre les propriétés communes régulées et les propriétés communes non régulées. Les propriétés communes non régulées sont protégées seulement par les restrictions portant sur le fait d'être membre ou non de la communauté, sans règle de conservation stricte, tandis que les propriétés communes régulées s'appuient à la fois sur une restriction de l'usage aux seuls membres et des règles d'usage appliquées aux membres (Ballet, 2007).

De surcroît, et malgré l'accès libre, déterminé par l'abondance de la ressource, accordé exclusivement aux membres de la tribu et aux ayants droit, les ressources communes restent loin d'être exploitées selon le laisser-faire. Les tribus usagères ont toujours confié aux conseils villageois et aux organisations traditionnelles locales la résolution des problèmes relatifs à l'extraction illégale de bois ou au pâturage non autorisé. Toute exploitation non conforme aux règles coutumières reconnues par la tribu est systématiquement combattue. Ses fonctions de jugement et de sanctions révèlent le sérieux et la rigueur quant à l'application des pratiques et des systèmes coutumiers. Ces derniers reflètent l'harmonie et l'homogénéité que les communautés indigènes entretiennent avec la nature sans exposer la ressource aux dangers du morcellement qu'implique la solution privative.

\section{Les structures locales: argument d'efficience en faveur de l'autochtonie}

Avant d'entamer les détails de cette hypothèse, nous rappelons que le raisonnement hardinien peut posséder sa marge de validité lorsqu'il s'agit d'une ressource au rythme de renouvellement assez long (exemple, les arbres), non placée sous l'égide d'une structure régulatrice, et surtout non soumise à un arsenal réglementaire (informel) susceptible de réguler les modalités d'accès et d'usage. Le berger, on le sait, est un agent économique (par nature) rationnel et dirigé par le désir de maximiser sa propre utilité en fonction de la contrainte qui s'impose à lui : la disponibilité du fourrage. Les partisans de cette hypothèse s'appuient sur un fondement théorique extrait dans sa majeure partie du dilemme du prisonnier issu de la théorie des jeux, de la théorie de la rationalité, ou encore des enseignements de l'École des droits de propriété. Mais, peut-on tirer des conclusions définitives de ce raisonnement basé sur la version substantielle de la rationalité ?

Les facteurs poussant l'individu à abandonner son individualisme et à s'engager pour le bien-être collectif ont attiré l'attention de plusieurs auteurs notamment les théoriciens du capital social (Bourdieu, 
2000), de la rationalité limitée (Kahneman, Tversky 1979 ; Tversky, Kahneman, 1974) et de l'action collective (Olson, 1956). Et c'est justement dans cette optique que, progressivement, la remise en cause de la gestion communautaire sera critiquée par plusieurs travaux empiriques menés au sein de l'anthropologie, de la sociologie voire même de l'économie dont les plus célèbres sont ceux menés par l'économiste américaine Elinor Ostrom qui lui ont valu son prix Nobel de 2009. En analysant ses conclusions, nous nous apercevons que les ressources possédées en commun par des groupements paysans ne sont pas forcément livrées à l'accès libre. Dans son célèbre ouvrage de 1990, elle a cité le cas de plusieurs communautés de type tribal qui sont pratiquement parvenues à éviter la tragédie des communaux, alors que les ressources possédées ne sont ni purement privées, ni étatiques. Il s'agit notamment des études effectuées sur des forêts communes en Suisse et au Japon, sur la gestion des nappes phréatiques en Californie, sur la répartition des eaux d'irrigation en Espagne, aux Philippines et au Sri Lanka, ou la gestion des pêcheries en Écosse et en Turquie.

Les réflexions d'Ostrom - ayant trait aux différents aspects de l'économie pastorale - ont trouvé leur succession dans les travaux empiriques menés par plusieurs chercheurs à l'instar de (Bazin et al., 2010). En s'appuyant sur une étude de terrain menée dans les forêts du HautAtlas marocain, ces derniers ont pu montrer la capacité autorégulatrice des paysans berbères - à travers leur groupement en structures locales - d'optimiser l'usage des pâturages communs. Ils ont expliqué comment ces structures informelles correspondent à un mécanisme régulateur compatible avec les objectifs de l'intérêt collectif. L'accès aux pâturages reste exclusivement accordé aux ayants-droit bien définis, mais dans la limite des règles et des normes que la structure locale impose. Un cadre réglementaire informel, extrait dans sa majeure partie de la coutume, a toujours permis le maintien d'un équilibre constant entre l'offre et la demande. Il repose principalement sur l'adoption d'une mise en défense saisonnière connue sous le nom de l'Agdal, qui consiste à fermer temporairement la zone déclarée comme dégradée afin d'assurer sa régénération. L'application de cette pratique a toujours permis le renouvellement du feuillage, l'accroissement des semis et le développement de la strate herbacée pendant les périodes de croissance de la végétation (Bourbouze, 2003). Le stock fourrager reste donc maintenu dans le temps.

Les réponses adaptatives à la diminution du fourrage sont multiples et peuvent varier d'une communauté à l'autre. Elles peuvent porter sur le droit d'accès comme elles peuvent concerner la réduction des tailles des troupeaux. L'examen des travaux menés dans cette optique nous permet d'observer « des formes d'ajustement dans les pratiques d'élevage. Cette question est traitée de manière assez détaillée dans les études consacrées à la Tanzanie et au Népal (Mascarenhas, Maganga, 1991 ; Shrestha, Uprety, 1991). Le déboisement, s'accompagnant d'une réduction de l'accès aux pacages, a obligé les ménages ruraux à modifier profondément leurs pratiques traditionnelles d'élevage dans les deux pays. Certaines familles sont obligées de réduire les effectifs de leur cheptel, malgré les effets négatifs sur les niveaux de subsistance et le revenu, tandis que d'autres doivent réévaluer les besoins de leurs bovins et l'avantage comparatif de l'élevage de certaines races bovines » (Ghimire, 1994).

Mais dans la plupart des cas, ce sont les Organisation traditionnelles locales qui constituent l'élément fondamental duquel les initiatives de gestion communautaire pastorale tirent leur efficience. Chaque organisation agit dans un rayon d'influence 
défini par les frontières territoriales de la tribu qu'elle représente. Cette particularité garantie le rapprochement du centre décisionnel des populations et permet de réguler toute action liée à l'activité du pâturage. Le contact permanent avec la ressource et avec les usagers qui en dépendent garantit à ces structures une parfaite connaissance du milieu ainsi qu'une meilleure prise en compte des réalités et des spécificités locales. De surcroît, cette proximité garantit une application efficiente des règles d'usage, une bonne définition des ayantsdroit ainsi qu'une surveillance optimale des zones dégradées ou mises en défense. Ces avantages ont toujours permis de lutter contre les modes d'exploitation inappropriés, et de limiter les comportements opportunistes du free riding.

$$
* *
$$$$
*
$$

L'exemple de Hardin illustre bien les incidences de l'accès libre et du laisser-faire, mais ces derniers ne sont pas nécessairement des caractéristiques intrinsèques de la propriété commune. Or, si la ressource peut disparaître à la suite de rivalités entre exploitants, elle peut également disparaître à la suite d'une décision prise volontairement par le propriétaire privé (exemple : transformation d'une surface forestière en terre agricole ou en zone d'habitation plus rentable que la vente du bois).

En outre, si le coût élevé de transaction est considéré comme facteur limitant le consensus entre les exploitants d'un pâturage commun, cette défaillance mise en avant par l'économiste américain Demsetz (1967) - ne semble pas épargner l'appropriation privative. Chaque berger a donc intérêt à assurer la fermeture et la surveillance de sa parcelle et en supporter le coût, ce qui était auparavant partagé par les tous membres. Car si l'on prend en compte l'égoïsme et la rivalité qui caractérisent les bergers suivant les propos de Hardin, chacun d'entre eux à donc intérêt à laisser son troupeau bénéficier de la parcelle de son voisin en passage clandestin. Platteau (2003) l'affirme explicitement en soulignant que « par rapport à la propriété communautaire, la propriété privée est plus coûteuse du point de vue des frais d'établissement et de protection que l'on peut qualifier de coûts directs de transaction ».

Le sens donné par Hardin à la propriété commune ignore toute possibilité de régulation de l'exploitation ainsi que toute celle d'exclusion. Alors que dans la plupart des cas, seuls les ayants-droit définis par la tribu usagère peuvent exploiter la ressource commune qui n'est pas forcément livrée à l'accès libre. L'exercice de ce droit d'exclusion pour le cas d'une ressource possédée par un groupe d'usagers nous permet de la considérer comme une propriété privée partagée, dont les caractéristiques sont loin d'être identiques à celles d'un bien public.

Les défaillances de la tradition hardinienne telles que nous venons de les expliquer ne permettent donc pas à la privatisation de se positionner comme solution adéquate. La mise en avant des limites qui peuvent être celles de la propriété commune ne signifie pas forcément que la propriété privée est irréprochable. Et ce n'est pas parce que les défaillances qui peuvent être celles de la propriété privée ne sont pas signalées par l'École des droits de propriété qu'elles n'existent pas.

L'auteur remercie son Directeur Monsieur JeanFrançois Noël pour son appui scientifique et reste entièrement responsable du contenu de l'article. 


\section{RÉFÉRENCES BIBLIOGRAPHIQUES}

Alchian A. A., Demsetz H. (1973). The property rights paradigm. Journal of Economic History, vol. $33, \mathrm{n}^{\circ} 1$, p. 16-27.

Anaya S. J. (1996). Indigenous Peoples in International Law. New York-Oxford, Oxford University Press.

Baland J.-M. et Platteau J.-P. (1996). Halting Degradation of Natural Resources: Is there a Role for Rural Communities? FAO, Oxford University Press. 423 p.

Ballet J. (2007). La gestion en commun des ressources naturelles : une perspective critique. Développement durable et territoires, Varia, http://developpementdurable.revues. org/3961

Bazin D., Bhukuth A., Roumane A. (2010). Organisations traditionnelles locales et capital social. Cas de la Jemaâ au Maroc en matière de gestion forestière. Revue Qualitique, septembre, $\mathrm{n}^{\circ} 218$.

Booth A.-L. (1998). Putting "forestry" and "community" into First Nations' resource management. Forestry Chronicle, vol. 74, $\mathrm{n}^{\circ} 3$, p. 347-351.

Bourbouze A. (2003). Stratégies des éleveurs et politiques forestières dans les montagnes méditerranéennes : du conflit à la conciliation. Rapport principal, session V1, Institut Agronomique Méditerranéen de Montpellier IAM CIHEAM, 13 p.

Bourdieu P. (2000). Les structures sociales de l'économie. Paris, Seuil, coll. « Liber», p. 289.

Brown D. (1999). Principles and Practice of Forest Co-management: Evidence from West-Central Africa. European Union Tropical Forestry Paper 2, Overseas Development Institute, London \& European Commission, Brussels, Belgique.

Buchy M., Hoverman S. (2000). Understanding public participation in forest planning: a review. Forest Policy and Economics, vol. 1, p. $15-25$.

Chouinard O., Perron J. (2002). Learning about community capacity in the Fundy Model Forest. Forestry Chronicle, vol. 78, $\mathrm{n}^{\circ} 5$, sept.-oct., p. 637-642.
Ciriacy-Wantrup S. V., Bishop R. C. (1975). Common property As a Concept in Natural Resources Policy. Natural Resources Journal, 15, p. 713-727.

Coase R.-H. (1960). The Problem of Social Cost. Journal of Law and Economics, vol. 3, p. 1-44.

Colfer C., Resosudarmo A. (2002). Which Way Forward? People, Forests, and Policymaking in Indonesia. Bogor, Indonesia, CIFOR's Publication, p. 433.

Dales J.-H. (1968). Pollution, Property and Prices, An Essay in Policy Making and Economics. Toronto, University of Toronto Press.

Demsetz H. (1967). Toward a Theory of Property Rights. American Economic Review, vol. 62, p. 347-359.

Ghimire K. B. (1994). Lutte contre le déboisement : analyse des initiatives locales. Revue internationale des forêts et des industries forestières (UNASYLVA), ${ }^{\circ} 178$, L'évolution du rôle des institutions forestières, vol. 45, 1994/3, FAO.

Gordon H.-S. (1954). The economic theory of a common property resource: the fishery. The Journal of Political Economy, $\mathrm{n}^{\circ} 62$, p. 124-142.

Hardin G. (1968). The tragedy of the commons. Science, $\mathrm{n}^{\circ}$ 162, p. 1243-1248.

Kahneman D., Tversky A. (1979). Prospect theory: an analysis of decision under risk. Econometrica, vol. 47, p. 263-291.

Kiss A. (1990). Wild Life Resources Management with Local Participation in Africa. World Bank Technical Paper, $\mathrm{n}^{\circ} 130$, Africa Department Series, Washington D.C.

Mc Kean M., Ostrom E. (1995). Régimes de propriété communautaire en forêt : simple vestige du passé ? Unasylva, vol. 46, n 180 , p. 3-15.

Olson M. (1956). The logic of collective action: Public Goods and the Theory of Groups. Cambridge, Harvard University Press, Logique de l'action collective (1978), Paris, Presses universitaires de France. 
Ostrom E. (1990). Governing the Commons: The Evolution of Institutions for Collective Action. New York, Cambridge University Press.

Philippe C. (2007). C'est trop tard pour la Terre. Paris, J.-C. Lattès, p. 176.

Pigou A.-C. (1920). The Economics of Welfare. New-York, Mac Millan.

Platteau J.-P. (2003). Droits de propriété et gestion efficace des ressources naturelles. Institut du développement durable et des relations internationales (IDDRI), Idées pour le débat, $n^{\circ} 15 / 2003$, p. 39.

Roumane A., Bhukuth A., Bazin D. (2010). La gestion des ressources forestières au Maroc: Une approche par le capital social. Working Papers $n^{\circ}$ 6-2010, Fonds pour la Recherche en Éthique Économique, http://ethique.perso.sfr.fr/FREECAHIERS.htm.

Samuelson P. A. (1954). The pure Theory of public Expenditure. The Review of Economics and Statistics, vol. 36, $\mathrm{n}^{\circ} 4$, p. 387-389.

Scott A. D. (1955). The fishery: the objectives of sole ownership. The Journal of Political Economy, n 63 , p. 116-124.
Tversky A., Kahneman D. (1974). Judgment under uncertainty: heuristics and biases. Science, $\mathrm{n}^{\circ} 185$, p. 1124-1131.

Varughese G., Ostrom E. (2001). The Contested Role of Heterogeneity in Collective Action: Evidence from Community Forestry in Nepal. World Development, vol. 29, $\mathrm{n}^{\circ} 5$, p. 747-765.

Vivien F.-D. (dir.) (2002). Biodiversité et appropriation : les droits de propriété en question. Natures Sciences Sociétés, Éditions Elsevier, p. 206.

Warming J. (1911). Om Grundrente af Fiskegrunde, Nationaløkonomisk Tidsskrift, 49, p. 499-505. Trad. in Andersen P. (1983), “On Rent of Fishing Grounds, History of Political Economy”, vol. 15, n 3, p. 391-396.

Wibe S. (1992). Défaillances des politiques dans la gestion des forêts. In OCDE, «Les défaillances du marché et des gouvernements dans la gestion de l'environnement. Les zones humides et les forêts ", Organisation de coopération et de développement économique, n $^{\circ}$ 89, p. 49-88. 